\title{
Pre-End-Stage Renal Disease Hemoglobin Variability Predicts Post-End-Stage Renal Disease Mortality in Patients Transitioning to Dialysis
}

\author{
Keiichi Sumida ${ }^{a-c}$ Charles Dyer Diskin ${ }^{a} \quad$ Miklos Z. Molnar ${ }^{a} d$ \\ Praveen K. Potukuchia Fridtjof Thomas ${ }^{\mathrm{e}}$ Jun Ling Lu ${ }^{\mathrm{a}}$ Connie M. Rhee \\ Elani Streja ${ }^{f}$ Kunihiro Yamagatac Kamyar Kalantar-Zadeh ${ }^{f}$ \\ Csaba P. Kovesdy ${ }^{a}$ g \\ ${ }^{a}$ Division of Nephrology, Department of Medicine, University of Tennessee Health Science Center, Memphis, TN, \\ USA; ${ }^{b}$ Nephrology Center, Toranomon Hospital Kajigaya, Kanagawa, Japan; ' Department of Nephrology, Faculty \\ of Medicine, University of Tsukuba, Ibaraki, Japan; ${ }^{d}$ Department of Transplantation and Surgery, Semmelweis

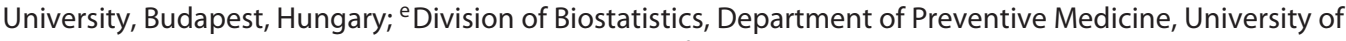 \\ Tennessee Health Science Center, Memphis, TN, USA; ${ }^{f}$ Harold Simmons Center for Chronic Disease Research \\ and Epidemiology, Division of Nephrology and Hypertension, University of California-Irvine, Orange, CA, USA; \\ gNephrology Section, Memphis VA Medical Center, Memphis, TN, USA
}

\author{
Keywords \\ Hemoglobin · Variability · Mortality · Chronic kidney \\ disease $\cdot$ End-stage renal disease $\cdot$ Transition
}

\begin{abstract}
Background: Hemoglobin variability (Hb-var) has been associated with increased mortality both in non-dialysis dependent chronic kidney disease (NDD-CKD) and end-stage renal disease (ESRD) patients. However, the impact of Hb-var in advanced NDD-CKD on outcomes after dialysis initiation remains unknown. Methods: Among 11,872 US veterans with advanced NDD-CKD transitioning to dialysis between October 2007 through September 2011, we assessed Hb-var calculated from the residual SD of at least $3 \mathrm{Hb}$ values during the last 6 months before dialysis initiation (prelude period) using within-subject linear regression models, and stratified into quartiles. Outcomes included post-transition all-cause, cardiovascular, and infection-related mortality, assessed in Cox proportional hazards models and adjusted for demo-
\end{abstract}

() 2017 S. Karger AG, Basel

E-Mail karger@karger.com www.karger.com/ajn graphics, comorbidities, length of hospitalization, medications, estimated glomerular filtration rate (eGFR), type of vascular access, $\mathrm{Hb}$ parameters (baseline $\mathrm{Hb}$ [i.e., intercept] and change in $\mathrm{Hb}$ [i.e., slope]), and number of $\mathrm{Hb}$ measurements. Results: Higher prelude $\mathrm{Hb}$-var was associated with use of iron and antiplatelet agents, tunneled dialysis catheter use, higher levels of baseline $\mathrm{Hb}$, change in $\mathrm{Hb}$, eGFR, and serum ferritin. After multivariable adjustment, higher prelude $\mathrm{Hb}$-var was associated with higher post-ESRD all-cause and infection-related mortality, but not cardiovascular mortality (adjusted hazard ratios [95\% Cl] for the highest [vs. lowest] quartile of Hb-var, 1.10 [1.02-1.19], 1.28 [0.93-1.75], and 0.93 [0.79-1.10], respectively). Conclusions: High pre-ESRD $\mathrm{Hb}$-var is associated with higher mortality, particularly from infectious causes rather than cardiovascular causes. Further research is required to clarify the underlying mechanisms and true causal nature of the observed association.

(c) 2017 S. Karger AG, Basel

K.S. and C.D.D. contributed equally to this work.
Csaba P. Kovesdy, MD

Nephrology Section, Memphis VA Medical Center

1030 Jefferson Avenue

Memphis, TN 38104 (USA)

E-Mail ckovesdy@uthsc.edu 


\section{Introduction}

Anemia is a frequent complication of chronic kidney disease (CKD) and is associated with worse outcomes in patients with CKD [1]. The introduction of erythropoiesis stimulating agents (ESAs) in 1989 has given clinicians the ability to increase hemoglobin $(\mathrm{Hb})$ levels, yet targeting normal $\mathrm{Hb}$ levels did not result in better survival, but rather in increased cardiovascular events and mortality in patients with CKD [2-4]. Maintenance of a stable $\mathrm{Hb}$ within the narrow range currently recommended in patients with CKD often requires frequent ESA dosing changes that, in addition to other factors such as age, intercurrent diseases, and iron therapy $[5,6]$, result in a continuous cycling of $\mathrm{Hb}$ levels in contrast to individuals with normal renal function whose $\mathrm{Hb}$ levels are held constant by oxygen delivery sensing feedback upon erythropoiesis. In recent years, observational studies have shown that higher $\mathrm{Hb}$ variability ( $\mathrm{Hb}$-var) is associated with adverse outcomes both in non-dialysis dependent CKD (NDD-CKD) and end-stage renal disease (ESRD) patients [7-16]. However, to the best of our knowledge, no previous studies have examined the impact of $\mathrm{Hb}$-var in late stage NDD-CKD on outcomes after dialysis initiation. Given the pervasive nature of anemia in this vulnerable population who experience the highest mortality immediately after the transition to dialysis and suffer from an exceptionally high health and economic burden $[1$, 17], the question whether high $\mathrm{Hb}$-var in the immediate pre-ESRD period is associated with post-transition mortality is of particular relevance. We therefore investigated the association of Hb-var in the pre-ESRD transition period with post-ESRD all-cause, cardiovascular, and infection-related mortality, using a large nationally representative cohort of US veterans with incident ESRD.

\section{Materials and Methods}

\section{Study Population}

We analyzed longitudinal data from the Transition of Care in CKD study, a retrospective cohort study examining US veterans with incident ESRD transitioning to dialysis from October 1, 2007, through September 30, 2011 [18-20]. A total of 52,172 US veterans were identified from the US Renal Data System (USRDS) [21] as a source population. The algorithm for the cohort definition is shown in online supplementary Figure 1 (for all online suppl. material, see www.karger.com/doi/10.1159/000484356). We extracted all $\mathrm{Hb}$ values measured during clinical encounters in any Veterans Affairs (VA) facility; patients without $\mathrm{Hb}$ measurements $(n=23,088)$ were excluded. We also excluded patients with less than $3 \mathrm{Hb}$ measurements recorded on different days within
6 months prior to dialysis initiation (i.e., 6-month "prelude period"; $n=17,068)$ and who were missing follow-up data $(n=144)$, resulting in an analytical sample of 11,872 patients.

\section{Exposure Variable}

The primary exposure of interest was Hb-var over the 6-month prelude period. Hb-var was defined as the residual SD calculated by within-subject linear regression models using all $\mathrm{Hb}$ values in each patient. We categorized the $\mathrm{Hb}$-var values into quartiles $(<0.46,0.46-<0.69,0.69-<0.96$, and $\geq 0.96 \mathrm{~g} / \mathrm{dL})$, using the lowest $\mathrm{Hb}$-var quartile as reference. The Hb-var level was also treated as a continuous variable to examine nonlinear associations by using a restricted cubic spline analysis. Baseline $\mathrm{Hb}$ level and average change in $\mathrm{Hb}(\mathrm{g} / \mathrm{dL}$ per month) were defined as the intercept and the slope estimated from the same regression models used to calculate $\mathrm{Hb}$-var.

\section{Covariates}

Data from the USRDS Patient and Medical Evidence files were used to determine patients' baseline demographic characteristics and type of vascular access at the time of dialysis initiation. Information on comorbidities was extracted from the VA Inpatient and Outpatient Medical SAS Datasets [22], using the International Classification of Diseases, Ninth Revision, Clinical Modification diagnostic and procedure codes and Current Procedural Terminology codes, as well as from VA/Centers for Medicare and Medicaid Services (CMS) data. The Charlson Comorbidity Index score was calculated using the Deyo modification for administrative datasets, without including kidney disease [23]. Cardiovascular disease was defined as the presence of diagnostic codes for angina, coronary artery disease, myocardial infarction, or cerebrovascular disease. Medication data were collected from both CMS Data (Medicare part D) and VA pharmacy dispensation records [24]. Patients who received at least 1 dispensation of medications within the 6-month prelude period were recorded as having been treated with these medications. Laboratory data were obtained from VA research databases as previously described $[25,26]$, and their baseline values were defined as the average of each covariate during the 6-month prelude period preceding dialysis initiation. Estimated glomerular filtration rate (eGFR) was calculated by the Chronic Kidney Disease Epidemiology Collaboration equation [27].

Outcome Assessment

The co-primary outcomes of interest were all-cause, cardiovascular, and infection-related mortality after dialysis initiation. The start of the follow-up period was the date of dialysis initiation, and patients were followed up until death or other censoring events including kidney transplantation, loss of follow-up, or the last date of available follow-up (December 27, 2012, and October 6, 2011, for all-cause and cause-specific mortality, respectively) [18-20]. All-cause mortality data, censoring events, and associated dates were obtained from VA and USRDS data sources [21]. Cause-specific mortality data were obtained from USRDS.

\section{Statistical Analysis}

Baseline patient characteristics were summarized according to Hb-var quartiles, and presented as number (percent) for categorical variables and the mean \pm SD for continuous variables with a normal distribution or median (interquartile intervals [IQI]) for those with a skewed distribution. Differences across 
quartiles were assessed using analysis of variance and chi-squared tests for continuous and categorical variables, respectively. We performed multivariable linear regression to identify variables independently associated with $\mathrm{Hb}$-var. Based on a priori knowledge and their availability in this study, the following explanatory variables were included: sociodemographics (age, sex, race, and marital status), comorbidities (diabetes mellitus, cardiovascular disease, congestive heart failure [CHF], peripheral vascular disease, lung disease, peptic ulcer disease, liver disease, malignancy, human immunodeficiency virus [HIV]/acquired immunodeficiency syndrome [AIDS]), Charlson comorbidity index, cumulative length of hospitalization during the 6-month prelude period, medications (ESAs, intravenous or oral iron, vitamin D analogs, angiotensin-converting enzyme inhibitors/angiotensin receptor blockers, antiplatelet agents, and warfarin), vascular access type, baseline $\mathrm{Hb}$, change in $\mathrm{Hb}$, number of $\mathrm{Hb}$ measurements over the 6-month prelude period, and eGFR and serum ferritin levels averaged over the 6 -month prelude period. Variance inflation factors were calculated to examine substantial multicollinearity among these parameters, and values $>5.0$ were considered to indicate collinearity. The association between $\mathrm{Hb}$-var and mortality was estimated using Cox proportional hazards models. Models were incrementally adjusted for the following potential confounders based on theoretical considerations: model 1 adjusted for age, sex, race/ethnicity, and marital status; model 2 additionally accounted for comorbidities (diabetes mellitus, cardiovascular disease, CHF, peripheral vascular disease, lung disease, peptic ulcer disease, liver disease, malignancy, and HIV/ AIDS), Charlson comorbidity index, and cumulative length of hospitalization during the 6-month prelude period as an indicator of sickness; model 3 additionally included medications (ESAs, intravenous or oral iron, vitamin D analogs, angiotensin-converting enzyme inhibitors s/ angiotensin receptor blockers, antiplatelet agents, and warfarin), eGFR, type of vascular access (arteriovenous fistula, arteriovenous graft, or catheter), baseline $\mathrm{Hb}$, change in $\mathrm{Hb}$, and number of $\mathrm{Hb}$ measurements over the 6-month prelude period. Tests for linear trend across quartiles were conducted by applying the median value of each quartile to relevant patients and modeling that variable as a continuous variable in the regression models. Restricted cubic spline models were used to investigate nonlinearity in fully adjusted associations between $\mathrm{Hb}$-var and mortality.

We performed several sensitivity analyses to evaluate the robustness of our main findings. The associations of $\mathrm{Hb}$-var with outcomes were examined in subgroups of patients stratified by age, race, prevalent diabetes mellitus, cardiovascular disease, and CHF, eGFR, use of ESAs and iron, and number of $\mathrm{Hb}$ measurements over the 6-month prelude period. Potential interactions were formally tested by including relevant interaction terms. We also investigated whether accounting for serum ferritin levels further attenuates the $\mathrm{Hb}$-var-mortality associations in the group of 8,605 patients with available serum ferritin measurements as an additional model (model 4 ). In order to account for the different $\mathrm{Hb}$ determinations between inpatient and outpatient settings, we further performed an additional analysis using at least 3 inpatient/outpatient $\mathrm{Hb}$ values separately to calculate the variability.

Compared to patients in the main cohort $(n=11,872)$, those who were excluded from the source cohort $(n=40,300)$ were older (71.6 vs. 66.0 years) and were less likely to be men (93.3 vs.
98.0\%), African-American (20.9 vs. $35.4 \%$ ), and diabetic (53.1 vs. $71.9 \%)$. Of the variables included in multivariable models, data points were missing for race $(0.2 \%)$, eGFR $(0.2 \%)$, vascular access type $(6.4 \%)$, and serum ferritin $(22.8 \%)$. Information about cause of death was also missing in 2,831 of the 5,711 (49.6\%) who died in our study population (online suppl. Table 1). Of the 11,872 patients in our study population, 11,088 (93.4\%) had complete data available for the main adjusted multivariable model (model 3 ). Due to the relatively low proportion of missing information in the main model, missing data was not imputed. The reported $p$ values are 2 -sided and reported as significant at $<0.05$ for all analyses. All analyses were conducted using STATA/MP version 14 (STATA Corporation, College Station, TX, USA). The study was approved by the Institutional Review Boards of the Memphis and Long Beach VA Medical Centers, with exemption from informed consent.

\section{Results}

\section{Baseline Characteristics}

Overall, the mean \pm SD age at baseline was $66.0 \pm 11.1$ years, $98.0 \%$ of patients were men, $35.4 \%$ were African American, and $71.9 \%$ were diabetic. The median (IQI) eGFR level was $13.1(9.9-17.7) \mathrm{mL} / \mathrm{min} / 1.73 \mathrm{~m}^{2}$. During the 6-month prelude period, patients had a median (IQI) of $8(5-15) \mathrm{Hb}$ measurements. The mean \pm SD prelude $\mathrm{Hb}$-var was $0.75 \pm 0.45 \mathrm{~g} / \mathrm{dL}$. Baseline characteristics in the overall cohort and stratified by $\mathrm{Hb}$-var quartiles are presented in Table 1. Compared to patients with lower $\mathrm{Hb}$-var, those with higher $\mathrm{Hb}$-var tended to have a poorer risk profile (higher prevalence of comorbidities, longer cumulative length of hospitalization, and lower serum albumin and higher serum ferritin levels) and had lower BMI and higher eGFR levels. They were also more likely to initiate dialysis therapy with a catheter and use ESAs, iron, and antiplatelet agents.

\section{Variables Associated with Prelude Hb-var}

Table 2 shows the association of $\mathrm{Hb}$-var with patient characteristics. After multivariable adjustment, history of peptic ulcer disease or malignancy, use of iron and antiplatelet agents, dialysis catheter use, and higher baseline $\mathrm{Hb}$, change in $\mathrm{Hb}$, number of $\mathrm{Hb}$ measurements, eGFR, and serum ferritin levels were associated with higher $\mathrm{Hb}$ var. In contrast, older age and use of warfarin were associated with lower $\mathrm{Hb}$-var.

\section{Association of Pre-ESRD Hb-var with Post-ESRD All-Cause Mortality}

There were 5,711 all-cause deaths during a median follow-up of 2.1 years (IQI 1.2-3.3 years; total time at risk, 
Table 1. Baseline patient characteristics according to quartiles of prelude $\mathrm{Hb}$ variability

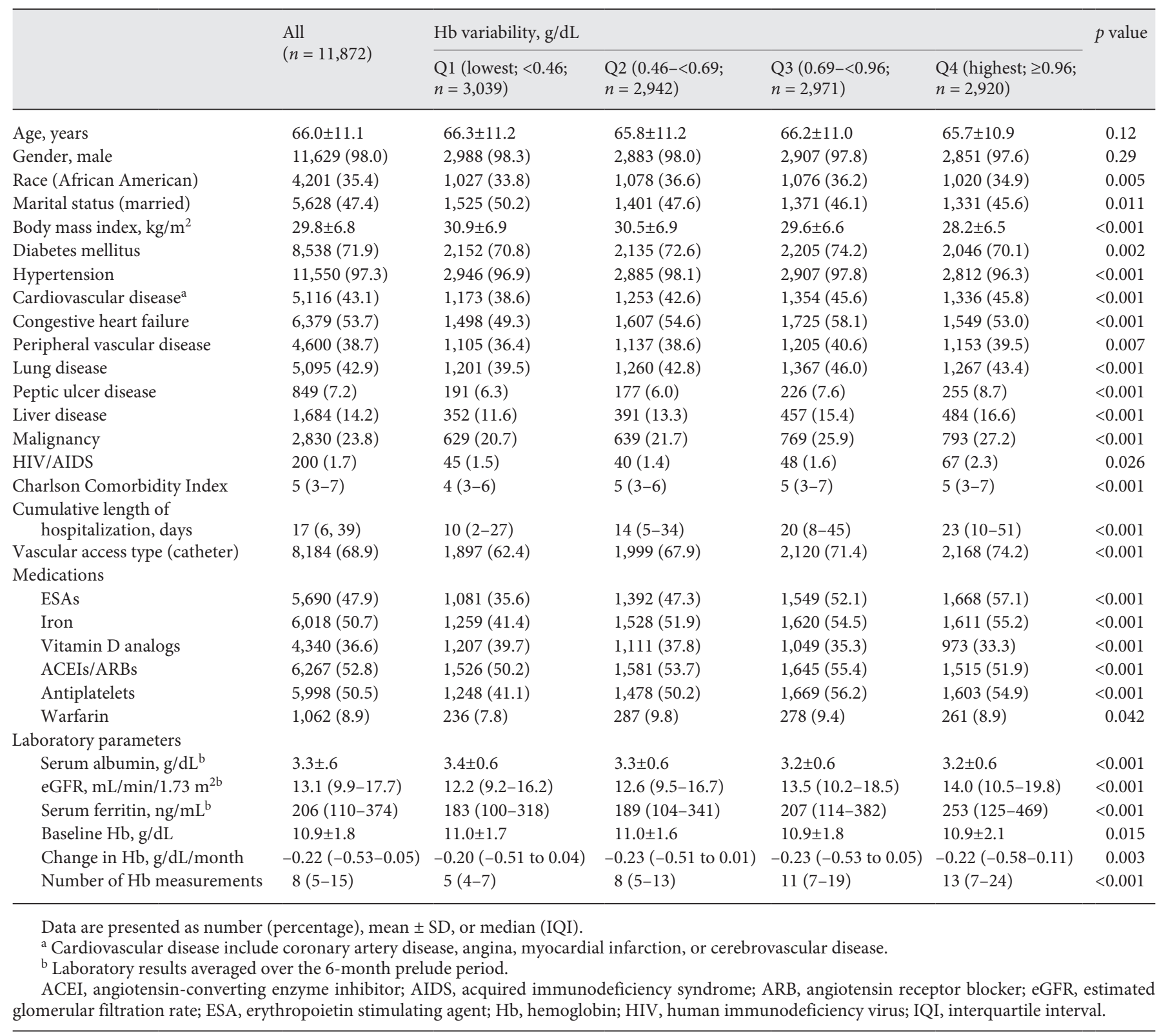

26,748 patient-years) following dialysis initiation (crude incidence rate, 213.5 per 1,000 patient-years; $95 \%$ CI 208.0-219.2). Table 3 shows the multivariable-adjusted hazard ratios (HRs) associated with pre-ESRD Hb-var quartiles. In the demographically adjusted model, higher $\mathrm{Hb}$-var quartiles were incrementally associated with higher all-cause mortality ( $p$ value for trend $<0.001$; Table $3)$. After further adjustment for other potential confounders, the association between $\mathrm{Hb}$-var and all-cause mortality was substantially attenuated but remained sta- tistically significant (adjusted HRs [95\% CI] for quartiles 2 through 4 [vs. quartile 1], 0.99 [0.91-1.07], 1.08 [1.001.17 ], and 1.10 [1.02-1.19], in model 3; $p$ value for trend $=$ 0.004; Table 3). As shown in Figure 1a, higher levels of $\mathrm{Hb}$-var treated as a continuous variable were monotonically associated with higher all-cause mortality.

In subgroup analyses, higher $\mathrm{Hb}$-var was associated with higher all-cause mortality across most subgroups (online suppl. Fig. 2). Race significantly modified the association of $\mathrm{Hb}$-var with all-cause mortality, with greater 
Table 2. Variables associated with prelude Hb variability

\begin{tabular}{|c|c|c|c|}
\hline Characteristics & Coefficient* & $95 \% \mathrm{CI}$ & $p$ value \\
\hline Age (per 1 year) & -0.001 & -0.002 to -0.0007 & 0.001 \\
\hline Gender (male vs. women) & 0.055 & -0.007 to 0.12 & 0.08 \\
\hline Race (African American vs. white) & -0.003 & -0.022 to 0.017 & 0.79 \\
\hline Marital status (married vs. non-married) & -0.007 & -0.025 to 0.01 & 0.42 \\
\hline \multicolumn{4}{|l|}{ Comorbidities (yes vs. no) } \\
\hline Congestive heart failure & 0.002 & -0.019 to 0.022 & 0.88 \\
\hline Peripheral vascular disease & 0.012 & 0.008 to 0.033 & 0.23 \\
\hline Lung disease & -0.008 & -0.028 to 0.012 & 0.42 \\
\hline Peptic ulcer disease & 0.047 & 0.012 to 0.082 & 0.008 \\
\hline Liver disease & 0.014 & -0.014 to 0.042 & 0.33 \\
\hline Malignancy & 0.030 & 0.003 to 0.058 & 0.031 \\
\hline ESAs & 0.094 & 0.075 to 0.113 & 0.27 \\
\hline Iron & 0.030 & 0.011 to 0.049 & 0.002 \\
\hline Vitamin D analogs & -0.014 & -0.032 to -0.005 & 0.16 \\
\hline ACEIs/ARBs & -0.009 & -0.027 to 0.009 & 0.30 \\
\hline Antiplatelet agents & 0.026 & 0.007 to 0.045 & 0.007 \\
\hline Warfarin & -0.052 & -0.085 to -0.020 & 0.002 \\
\hline Vascular access type (catheter vs. others) & 0.032 & 0.012 to 0.052 & 0.002 \\
\hline \multicolumn{4}{|l|}{ Laboratory parameters } \\
\hline Baseline $\mathrm{Hb}$ (per $1 \mathrm{~g} / \mathrm{dL}$ ) & 0.014 & 0.008 to 0.019 & 0.002 \\
\hline Change in $\mathrm{Hb}$ (per $1 \mathrm{~g} / \mathrm{dL} /$ month) & 0.0004 & 0.0003 to 0.0007 & $<0.001$ \\
\hline Number of $\mathrm{Hb}$ measurements & 0.006 & 0.005 to 0.006 & $<0.001$ \\
\hline eGFR $\left(\text { per } 1 \mathrm{~mL} / \mathrm{min} / 1.73 \mathrm{~m}^{2}\right)^{\mathrm{b}}$ & 0.002 & 0.0009 to 0.003 & $<0.001$ \\
\hline
\end{tabular}

* Coefficient for multivariable linear regression models. Value of coefficient represents change in $\mathrm{Hb}$ variability (g/dL) per 1 unit change in each factor. Positive and negative numbers indicate higher and lower Hb variability per 1 unit change in factors, respectively. The variance inflation factors of these parameters were all less than 5.

${ }^{a}$ Cardiovascular disease include coronary artery disease, angina, myocardial infarction, or cerebrovascular disease.

${ }^{b}$ Laboratory results averaged over the 6-month prelude period.

ACEI, angiotensin-converting enzyme inhibitor; AIDS, acquired immunodeficiency syndrome; ARB, angiotensin receptor blocker; eGFR, estimated glomerular filtration rate; ESA, erythropoietin stimulating agent; Hb, hemoglobin; HIV, human immunodeficiency virus.

association of higher Hb-var with all-cause mortality among African-Americans. Results were similar after further adjustment for serum ferritin level and using inpatient or outpatient $\mathrm{Hb}$ values separately to calculate the variability, albeit with borderline significance (online suppl. Tables 2a-4a).

\section{Association of Pre-ESRD Hb-var with Post-ESRD}

Cardiovascular and Infection-Related Mortality

During a median follow-up of 1.3 years (IQI 0.6-2.3 years) following dialysis initiation, 1,045 and 382 deaths occurred from cardiovascular and infection-related causes, respectively. In the demographically adjusted model, Hb-var quartiles were incrementally associated with cardiovascular mortality, with significantly higher death risks seen in higher $\mathrm{Hb}$-var quartiles. This association was considerably attenuated and no longer significant after further adjustment for other potential confounders (adjusted HRs [95\% CI] for $\mathrm{Hb}$-var quartiles 2 through 4 [vs. quartile 1], 0.92 [0.79-1.10], 1.03 [0.881.21 ], and 0.93 [0.79-1.10], in model 3; $p$ value for trend $=$ 0.63; Table 4). In contrast, higher Hb-var quartiles were 


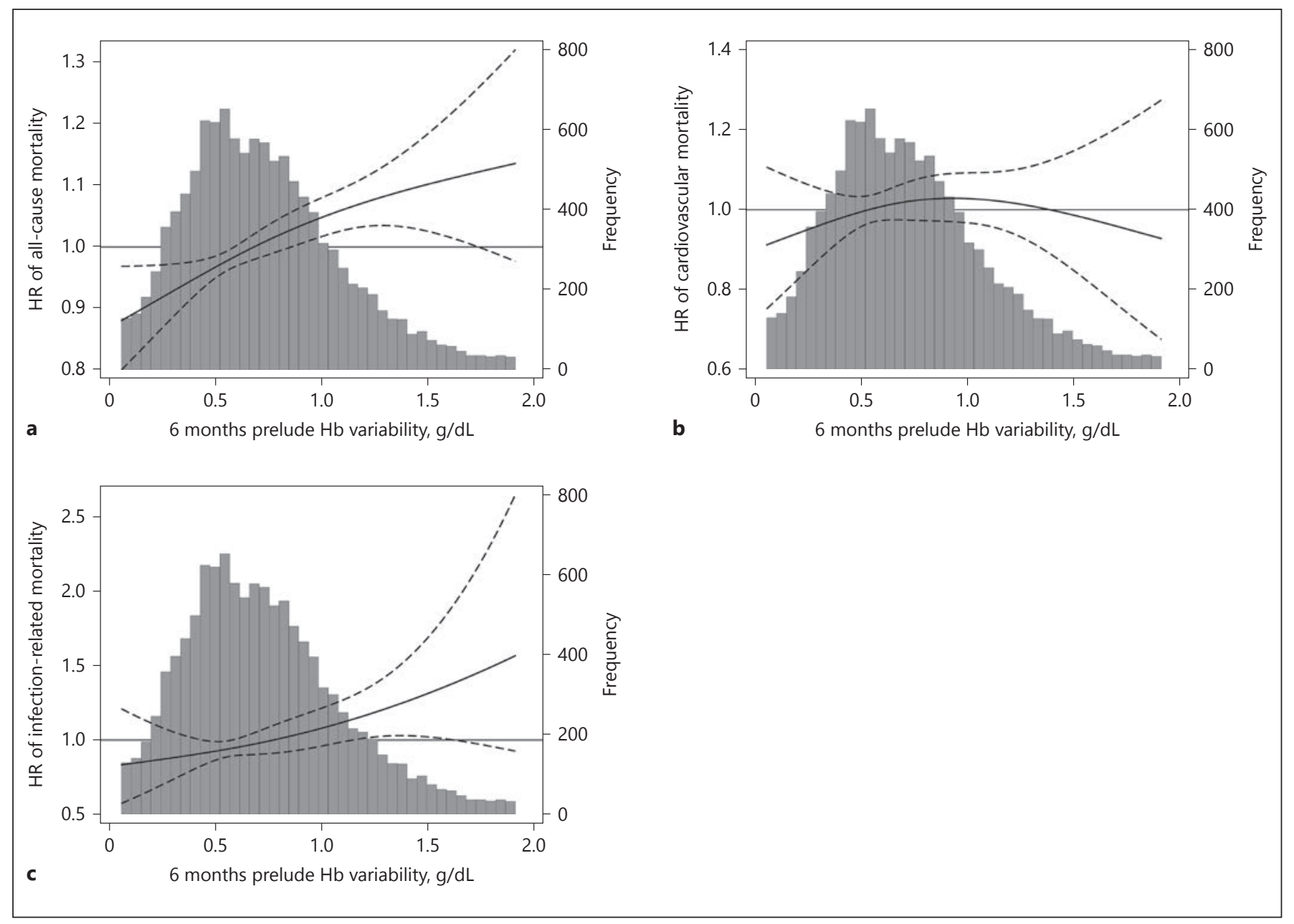

Fig. 1. Association of prelude $\mathrm{Hb}$ variability with (a) all-cause, (b) cardiovascular, and (c) infection-related mortality after dialysis initiation Solid and dashed lines represent HR and 95\% CI, respectively. A hazard reference ratio of 1 (solid horizontal line) and a histogram of observed $\mathrm{Hb}$ variability values are overlaid. The $\mathrm{x}$ axis shows $\mathrm{Hb}$ variability levels, trimmed at 2 and $98 \%$. Model is adjusted for age, sex, race/ethnicity, marital status, comorbidities (diabetes mellitus, cardiovascular disease, congestive heart failure, peripheral vascular disease, lung disease, peptic ulcer disease, liver disease, malignancy, and HIV/AIDS), Charlson comorbidity index, cumulative length of hospitalization, medications (ESAs, in-

associated with a trend towards higher infection-related mortality in the fully adjusted multivariable model (adjusted HRs [95\% CI] for Hb-var quartiles 2 through 4 [vs. quartile 1], 1.02 [0.74-1.41], 1.19 [0.87-1.63], and 1.28 [0.93-1.75], in model 3; $p$ value for trend $=0.079$; Table 4). When using restricted cubic spline models, there was a weak but nonsignificant reverse U-shaped association of Hb-var with cardiovascular mortality (Fig. 1b); while the pattern of association between $\mathrm{Hb}$-var and infection- travenous or oral iron, vitamin D analogs, ACEIs/ARBs, antiplatelets, and warfarin), eGFR levels averaged over the 6-month prelude period, type of vascular access (arteriovenous fistula, arteriovenous graft, or catheter), $\mathrm{Hb}$ variability parameters (baseline $\mathrm{Hb}$ level and change in $\mathrm{Hb}$ ), and number of $\mathrm{Hb}$ measurements during the 6-month prelude period. ACEI, angiotensin-converting enzyme inhibitor; AIDS, acquired immunodeficiency syndrome; $\mathrm{ARB}$, angiotensin receptor blocker; eGFR, estimated glomerular filtration rate; ESA, erythropoietin stimulating agent; $\mathrm{Hb}$, hemoglobin; HIV, human immunodeficiency virus.

related mortality was qualitatively similar to that with allcause mortality, with higher mortality seen in those with higher Hb-var (Fig. 1c).

In subgroup analyses, the pattern of associations of $\mathrm{Hb}$-var with cardiovascular and infection-related mortality were generally consistent across selected subgroups (online suppl. Fig. 1b, c), with a few exceptions: the risk of infection-related mortality associated with higher $\mathrm{Hb}$ var was greater in patients with $\mathrm{eGFR}<15 \mathrm{~mL} / \mathrm{min} / 1.73 \mathrm{~m}^{2}$ 
Table 3. Adjusted hazard ratios ( $95 \%$ CI) for all-cause mortality after dialysis initiation by Hb variability quartiles over the 6-month prelude period

\begin{tabular}{|c|c|c|c|c|c|}
\hline & \multicolumn{4}{|c|}{ Quartile of prelude $\mathrm{Hb}$ variability, g/dL } & $p$ for trend \\
\hline Events & $1,323(43.5)$ & $1,290(43.8)$ & $1,521(51.2)$ & $1,577(54.0)$ & \\
\hline Model 1 & 1 (reference) & $1.05(0.97-1.13)$ & $1.29(1.20-1.39)$ & $1.37(1.28-1.48)$ & $<0.001$ \\
\hline Model 2 & 1 (reference) & $0.99(0.92-1.07)$ & $1.17(1.08-1.26)$ & $1.23(1.14-1.32)$ & $<0.001$ \\
\hline
\end{tabular}

${ }^{a}$ Linear trend across the quartiles using the median $\mathrm{Hb}$ variability value of each quartile.

Data are presented as number (percentage) or hazard ratio (95\% CI) unless otherwise specified. Models are as follows: model 1 is adjusted for age, sex, race/ethnicity, and marital status; model 2 is adjusted for the variables in model 1 plus comorbidities (diabetes mellitus, cardiovascular disease, congestive heart failure, peripheral vascular disease, lung disease, peptic ulcer disease, liver disease, malignancy, and HIV/AIDS), Charlson comorbidity index, and cumulative length of hospitalization; and model 3 is adjusted for the variables in model 2 plus medications (ESAs, intravenous or oral iron, vitamin D analogs, ACEIs/ARBs, antiplatelets, and warfarin), eGFR levels averaged over the six-month prelude period, type of vascular access (arteriovenous fistula, arteriovenous graft, or catheter), Hb variability parameters (baseline $\mathrm{Hb}$ level and change in $\mathrm{Hb}$ ), and number of $\mathrm{Hb}$ measurements during the 6-month prelude period.

ACEI, angiotensin-converting enzyme inhibitor; AIDS, acquired immunodeficiency syndrome; ARB, angiotensin receptor blocker; eGFR, estimated glomerular filtration rate; ESA, erythropoietin stimulating agent; Hb, hemoglobin; HIV, human immunodeficiency virus.

and those treated with ESAs, with statistically significant interactions. The associations were robust to additional adjustment for serum ferritin levels and when using inpatient or outpatient $\mathrm{Hb}$ values separately to calculate the variability (online suppl. Tables $2 \mathrm{~b}-4 \mathrm{c}$ ).

\section{Discussion}

In this large national cohort of 11,872 US veterans with advanced NDD-CKD transitioning to dialysis, we found that higher $\mathrm{Hb}$-var was independently associated with higher all-cause and infection-related mortality, but not cardiovascular mortality, following dialysis initiation. Compared with patients in the lowest $\mathrm{Hb}$-var quartile $(<0.46 \mathrm{~g} / \mathrm{dL})$, those in the highest $\mathrm{Hb}$-var quartile $(\geq 0.96 \mathrm{~g} / \mathrm{dL})$ had 10 and $28 \%$ higher all-cause and infection-related mortality, respectively, after adjusting for various potential confounders. During the 6-month prelude period, we also found that history of peptic ulcer disease or malignancy, use of iron and antiplatelet agents, dialysis catheter use, and higher number of $\mathrm{Hb}$ measurements and levels of baseline $\mathrm{Hb}$, change in $\mathrm{Hb}$, eGFR, and serum ferritin were all associated with higher Hb-var.

These results are similar to some aspects of several previous studies that reported the association of greater
Hb-var with poor survival, almost exclusively in patients undergoing maintenance hemodialysis [9-16]. Recently, a few observational studies have also demonstrated a similar Hb-var-mortality association in NDDCKD populations $[7,8]$, suggesting its prognostic implications throughout different stages of CKD. Most importantly, however, no previous studies have examined the Hb-var-mortality association during the ESRD transition period. The extremely high mortality experienced by incident ESRD patients immediately following transition to dialysis [17] makes the assessment of modifiable pre-transition risk factors (such as $\mathrm{Hb}$-var, and others) especially important in order to find treatment targets that might have a positive effect on their survival.

Several possible explanations have been proposed for the underlying mechanisms of high $\mathrm{Hb}$-var, such as differences in pharmacokinetic and bioavailability parameters among drugs that modulate $\mathrm{Hb}$ synthesis (e.g., ESAs and iron) [28, 29], a high number of comorbidities, intercurrent events, and hospitalizations [9], anemia management practice patterns, and reimbursement policies [30]. In line with these findings, we identified several clinical factors associated with higher prelude $\mathrm{Hb}$ var, including history of peptic ulcer disease or malignancy, use of iron and antiplatelet agents, dialysis catheter use, and higher number of $\mathrm{Hb}$ measurements 
Table 4. Adjusted hazard ratios $(95 \% \mathrm{CI})$ for cardiovascular and infection-related mortality after dialysis initiation by $\mathrm{Hb}$ variability quartiles over the 6-month prelude period

\begin{tabular}{|c|c|c|c|c|c|}
\hline & \multicolumn{4}{|c|}{ Quartile of prelude $\mathrm{Hb}$ variability } & $p$ for trend \\
\hline Events & $333(11.0)$ & $322(10.9)$ & $389(13.1)$ & $361(12.4)$ & \\
\hline Model 1 & 1 (reference) & $1.03(0.88-1.20)$ & $1.28(1.11-1.49)$ & $1.20(1.04-1.40)$ & 0.003 \\
\hline \multicolumn{6}{|l|}{ Infection-related } \\
\hline Events & $77(2.5)$ & $81(2.8)$ & $105(3.5)$ & $119(4.1)$ & \\
\hline Model 1 & 1 (reference) & $1.11(0.81-1.51)$ & $1.46(1.09-1.96)$ & $1.71(1.28-2.28)$ & $<0.001$ \\
\hline Model 2 & 1 (reference) & $1.07(0.78-1.46)$ & $1.36(1.00-1.82)$ & $1.53(1.15-2.05)$ & 0.001 \\
\hline Model 3 & 1 (reference) & $1.02(0.74-1.41)$ & $1.19(0.87-1.63)$ & $1.28(0.93-1.75)$ & 0.079 \\
\hline
\end{tabular}

${ }^{a}$ Linear trend across the quartiles using the median $\mathrm{Hb}$ variability value of each quartile.

Data are presented as number (\%) or hazard ratio (95\% CI) unless otherwise specified. Models are as follows: model 1is adjusted for age, gender, race/ethnicity, and marital status; model 2 is adjusted for the variables in model 1 plus comorbidities (diabetes mellitus, cardiovascular disease, congestive heart failure, peripheral vascular disease, lung disease, peptic ulcer disease, liver disease, malignancy, and HIV/AIDS), Charlson comorbidity index, and cumulative length of hospitalization; and model 3 is adjusted for the variables in model 2 plus medications (ESAs, intravenous or oral iron, vitamin D analogs, ACEIs/ARBs, antiplatelets, and warfarin), eGFR levels averaged over the 6-month prelude period, type of vascular access (arteriovenous fistula, arteriovenous graft, or catheter), Hb variability parameters (baseline $\mathrm{Hb}$ level and change in $\mathrm{Hb}$ ), and number of $\mathrm{Hb}$ measurements during the 6-month prelude period.

ACEI, angiotensin-converting enzyme inhibitor; AIDS, acquired immunodeficiency syndrome; ARB, angiotensin receptor blocker; eGFR, estimated glomerular filtration rate; ESA, erythropoietin stimulating agent; Hb, hemoglobin; HIV, human immunodeficiency virus.

and levels of baseline $\mathrm{Hb}$, change in $\mathrm{Hb}$, eGFR, and serum ferritin. The substantial attenuation observed in our mortality risk estimates after accounting for all of these factors may, in turn, support their potential involvement as underlying pathophysiological mechanisms in the $\mathrm{Hb}$-var-mortality relationship. In fact, patients with advanced NDD-CKD have an exceptionally high burden of numerous comorbidities, and higher prelude $\mathrm{Hb}$-var may merely be a marker of more severe underlying comorbid conditions with concomitant chronic anemia requiring iron and/or ESAs supplementation, which could consequently contribute to the higher risk of all-cause mortality.

It has been suggested that increased $\mathrm{Hb}$-var can also serve as a potential direct mediator of organ dysfunction or injury by repeated episodes of relative ischemia to vital organs and $\mathrm{Hb}$ overshoot, the former causing left ventricular dilation or hypertrophy through the development of pathologic changes of myocardium [31, 32], the latter resulting in elevated blood pressure with risk for hypertensive encephalopathy [33], increased thrombotic events [2], and accelerated left ventricular dysfunction and hypertrophy [34], mostly in patients receiving hemodialysis. In the range of $\mathrm{Hb}$ levels typically encountered in the NDD-CKD population, anemia as a cause of solid organ dysfunction mediated by ischemia would be unusual, which could explain why we observed less contributions of prelude $\mathrm{Hb}$-var to the risk of cardiovascular mortality than infection-related mortality following dialysis initiation. This seemingly counterintuitive observation might also be partly explained by survivorship bias in this unique study population, such that patients who had suffered from the abovementioned cardiovascular complications and had higher $\mathrm{Hb}$-var may have died before reaching ESRD.

Meanwhile, our findings on the association between $\mathrm{Hb}$-var and infection-related mortality are generally consistent with a few previous studies that investigated the relationship between $\mathrm{Hb}$-var and incidence of infectious diseases $[9,16]$. Although we cannot infer a causal relationship from observational studies, there are some plausible explanations linking higher $\mathrm{Hb}$-var to the risk of infection. In the present study, despite almost identical levels of baseline $\mathrm{Hb}$ across $\mathrm{Hb}$-var quartiles, we observed 
higher serum ferritin levels and a higher prevalence of patients who used iron and ESAs in higher (vs. lower) Hbvar quartiles, which may have reflected underlying systemic inflammation due to infections among patients with higher $\mathrm{Hb}$-var. Increase in systemic inflammation may inhibit erythropoiesis and result in a blunted or absent response to ESAs despite its high-dose administration. This ESA hyporesponsiveness, which itself is also an independent risk factor for poor survival [35], may trigger the use of more iron because of the low transferrin saturation levels which result from inflammatory blockade of reticuloendothelial stores of iron [36]. Iron administration is highly effective in replenishing iron stores to accelerate erythropoiesis, but its excessive use may in turn result in impaired host innate immune response [37, 38]. Indeed, iron overload has been shown to promote apoptosis of helper $\mathrm{CD} 4^{+} \mathrm{T}$ lymphocytes through iron-induced oxidative stress $[39,40]$ and impair phagocytic and bactericidal capacities of polymorphonuclear leukocytes [41-43]; thereby increasing susceptibility to infections. These underlying mechanisms could synergistically contribute to a higher risk of infection-related mortality. In our study, however, the association of $\mathrm{Hb}$-var with infection-related mortality still remained statistically significant even after accounting for serum ferritin levels and use of ESAs and iron as well as various potential confounders, suggesting that higher prelude $\mathrm{Hb}$-var could be a harbinger of future deaths from infectious causes independent of known risk factors. Given the considerable uncertainties about the physiological mechanism of $\mathrm{Hb}$ var and the optimal approach to anemia management in the transition period, the effect of such interventions toward reducing $\mathrm{Hb}$-var on patient outcomes may deserve further investigation.

Our study results must be interpreted in light of several limitations. First, our cohort consisted predominantly of male US veterans; hence, generalization of the results to women or patients from other geographical areas needs to be undertaken cautiously. Second, the effect of longitudinal changes in Hb-var and other potential confounders such as ESA use and iron status over the post-ESRD follow-up period was not accounted for; therefore, it is possible that such time-dependent factors might affect the observed associations. However, given the unique nature of this study which examined the impact of pre-ESRD $\mathrm{Hb}$-var on postESRD outcomes, the observed results with the use of fixed prelude baseline covariates would still be of value, providing potential prognostic implications for postESRD outcomes in patients with advanced NDD-CKD.
Finally, as with all observational studies, we cannot eliminate the possibility of unmeasured confounders such as proteinuria.

In conclusion, in this large national cohort of US veterans with advanced NDD-CKD transitioning to dialysis, a greater pre-ESRD Hb-var was associated with higher post-ESRD mortality, especially with infection-related mortality. Our findings broaden the recognition of the prognostic importance of $\mathrm{Hb}$-var in the immediate preESRD period on post-ESRD outcomes and suggest the need for careful attention to high Hb-var in CKD patients during the transition period. Further research is required to clarify the underlying mechanisms and to test whether modification of pre-ESRD Hb-var can improve clinical outcomes in incident ESRD patients.

\section{Acknowledgments}

This study is supported by grant 5U01DK102163 from the National Institute of Health (NIH) to K.K.-Z. and C.P.K., and by resources from the US Department of Veterans Affairs. The data reported here have been supplied in part by the United States Renal Data System (USRDS). Support for VA/CMS data is provided by the Department of Veterans Affairs, Veterans Health Administration, Office of Research and Development, Health Services Research and Development, VA Information Resource Center (project numbers SDR 02-237 and 98-004).

Drs. Csaba P. Kovesdy and Kamyar Kalantar-Zadeh are employees of the Department of Veterans Affairs. The interpretation and reporting of these data are the responsibility of the authors and in no way should be seen as official policy or interpretation of the Department of Veterans Affairs or the US government. The results of this paper have not been published previously in whole or part.

\section{Disclosure Statement}

None of the authors have relevant conflicts of interest.

References

1 KDIGO clinical practice guideline for anemia in chronic kidney disease. Kidney Int Suppl 2012;2:279-335.

2 Singh AK, Szczech L, Tang KL, Barnhart H, Sapp S, Wolfson M, Reddan D; Investigators C: Correction of anemia with epoetin alfa in chronic kidney disease. N Engl J Med 2006; 355:2085-2098.

3 Drueke TB, Locatelli F, Clyne N, Eckardt KU, Macdougall IC, Tsakiris D, Burger HU, Scherhag A; CREATE Investigators: Normalization of hemoglobin level in patients with chronic kidney disease and anemia. N Engl J Med 2006;355:2071-2084. 
4 Pfeffer MA, Burdmann EA, Chen CY, Cooper ME, de Zeeuw D, Eckardt KU, Feyzi JM, Ivanovich $\mathrm{P}$, Kewalramani R, Levey AS, Lewis EF, McGill JB, McMurray JJ, Parfrey P, Parving HH, Remuzzi G, Singh AK, Solomon SD, Toto R, TREAT Investigators: A trial of darbepoetin alfa in type 2 diabetes and chronic kidney disease. N Engl J Med 2009;361:20192032.

5 Gupta AK, David W: Does erythropoietin cause hemoglobin variability - is it "normal"? PLoS One 2014;9:e92890.

6 Arikan H, Asicioglu E, Velioglu A, Nalcaci S, Birdal G, Guler D, Koc M, Tuglular S, Ozener C: Determinants of hemoglobin variability in stable peritoneal dialysis patients. Int Urol Nephrol 2014;46:1427-1434.

7 Boudville NC, Djurdjev O, Macdougall IC, de Francisco AL, Deray G, Besarab A, Stevens $P E$, Walker $R G$, Urena $P$, Inigo $P$, Minutolo R, Haviv YS, Yeates K, Aguera ML, MacRae JM, Levin A: Hemoglobin variability in nondialysis chronic kidney disease: examining the association with mortality. Clin J Am Soc Nephrol 2009;4:11761182.

8 Szeto CC, Kwan BC, Chow KM, Pang WF, Leung CB, Li PK: Haemoglobin variability in chinese pre-dialysis CKD patients not receiving erythropoietin. Nephrol Dial Transplant 2011;26:2919-2924.

9 Ebben JP, Gilbertson DT, Foley RN, Collins AJ: Hemoglobin level variability: associations with comorbidity, intercurrent events, and hospitalizations. Clin J Am Soc Nephrol 2006; 1:1205-1210.

10 Yang W, Israni RK, Brunelli SM, Joffe MM, Fishbane S, Feldman HI: Hemoglobin variability and mortality in ESRD. J Am Soc Nephrol 2007;18:3164-3170.

11 Gilbertson DT, Ebben JP, Foley RN, Weinhandl ED, Bradbury BD, Collins AJ: Hemoglobin level variability: associations with mortality. Clin J Am Soc Nephrol 2008;3: 133-138.

12 Brunelli SM, Lynch KE, Ankers ED, Joffe MM, Yang W, Thadhani RI, Feldman HI: Association of hemoglobin variability and mortality among contemporary incident hemodialysis patients. Clin J Am Soc Nephrol 2008;3: 1733-1740.

13 Brunelli SM, Joffe MM, Israni RK, Yang W, Fishbane S, Berns JS, Feldman HI: Historyadjusted marginal structural analysis of the association between hemoglobin variability and mortality among chronic hemodialysis patients. Clin J Am Soc Nephrol 2008;3:777782.

14 Pisoni RL, Bragg-Gresham JL, Fuller DS, Morgenstern H, Canaud B, Locatelli F, Li Y, Gillespie B, Wolfe RA, Port FK, Robinson BM: Facility-level interpatient hemoglobin variability in hemodialysis centers participating in the dialysis outcomes and practice patterns study (DOPPS): associations with mortality, patient characteristics, and facility practices. Am J Kidney Dis 2011;57:266-275.
15 Weinhandl ED, Peng Y, Gilbertson DT, Bradbury BD, Collins AJ: Hemoglobin variability and mortality: confounding by disease severity. Am J Kidney Dis 2011;57:255265.

16 Kuragano T, Matsumura O, Matsuda A, Hara T, Kiyomoto H, Murata T, Kitamura K, Fujimoto S, Hase H, Joki N, Fukatsu A, Inoue T, Itakura I, Nakanishi T: Association between hemoglobin variability, serum ferritin levels, and adverse events/mortality in maintenance hemodialysis patients. Kidney Int 2014;86: 845-854.

17 Saran R, Li Y, Robinson B, Abbott KC, Agodoa LY, Ayanian J, Bragg-Gresham J, Balkrishnan R, Chen JL, Cope E, Eggers PW, Gillen D, Gipson D, Hailpern SM, Hall YN, He K, Herman W, Heung M, Hirth RA, Hutton D, Jacobsen SJ, Kalantar-Zadeh K, Kovesdy CP, Lu Y, Molnar MZ, Morgenstern H, Nallamothu B, Nguyen DV, O'Hare AM, Plattner B, Pisoni R, Port FK, Rao P, Rhee CM, Sakhuja A, Schaubel DE, Selewski DT, Shahinian V, Sim JJ, Song P, Streja E, Kurella Tamura M, Tentori F, White S, Woodside K, Hirth RA: Us renal data system 2015 annual data report: epidemiology of kidney disease in the united states. Am J Kidney Dis 2016; 67:A7-A8.

18 Sumida K, Molnar MZ, Potukuchi PK, Thomas F, Lu JL, Jing J, Ravel VA, Soohoo M, Rhee CM, Streja E, Kalantar-Zadeh K, Kovesdy CP: Association of slopes of estimated glomerular filtration rate with postend-stage renal disease mortality in patients with advanced chronic kidney disease transitioning to dialysis. Mayo Clin Proc 2016; 91:196-207.

19 Sumida K, Molnar MZ, Potukuchi PK, Thomas F, Lu JL, Ravel VA, Soohoo M, Rhee $\mathrm{CM}$, Streja E, Yamagata K, Kalantar-Zadeh $\mathrm{K}$, Kovesdy CP: Association between vascular access creation and deceleration of estimated glomerular filtration rate decline in late-stage chronic kidney disease patients transitioning to end-stage renal disease. Nephrol Dial Transplant 2017;32:13301337.

20 Molnar MZ, Gosmanova EO, Sumida K, Potukuchi PK, Lu JL, Jing J, Ravel VA, Soohoo M, Rhee CM, Streja E, Kalantar-Zadeh K, Kovesdy CP: Predialysis cardiovascular disease medication adherence and mortality after transition to dialysis. Am J Kidney Dis 2016;68:609-618.

21 United States Renal Data System, 2014 Annual Data Report: Epidemiology of Kidney Disease in the United States. Bethesda, National Institutes of Health, National Institute of Diabetes and Digestive and Kidney Diseases, 2014

22 US Department of Veterans Affairs: VIReC Research User Guide; VHA Medical SAS Inpatient Datasets FY2006-2007. Hines, VA Information Resource Center, 2007.

23 Deyo RA, Cherkin DC, Ciol MA: Adapting a clinical comorbidity index for use with ICD-
9-CM administrative databases. J Clin Epidemiol 1992;45:613-619.

24 VA Information Resource Center (VIReC). VIReC Research User Guide: VHA Pharmacy Prescription Data, ed 2. US Department of Veterans Affairs, Health Services Research and Development Service. Hines, VA Information Resource Center, 2008.

25 Kovesdy CP, Norris KC, Boulware LE, Lu JL, Ma JZ, Streja E, Molnar MZ, Kalantar-Zadeh K: Association of race with mortality and cardiovascular events in a large cohort of US veterans. Circulation 2015;132:15381548.

26 Kovesdy CP, Alrifai A, Gosmanova EO, Lu JL, Canada RB, Wall BM, Hung AM, Molnar MZ, Kalantar-Zadeh K: Age and outcomes associated with BP in patients with incident CKD. Clin J Am Soc Nephrol 2016;11:821831

27 Levey AS, Stevens LA, Schmid CH, Zhang YL, Castro AF 3rd, Feldman HI, Kusek JW, Eggers P, Van Lente F, Greene T, Coresh J; CKDEPI (Chronic Kidney Disease Epidemiology Collaboration): A new equation to estimate glomerular filtration rate. Ann Intern Med 2009;150:604-612.

28 Aronoff GR: Safety of intravenous iron in clinical practice: implications for anemia management protocols. J Am Soc Nephrol 2004;15(suppl 2):S99-S106.

29 Kalantar-Zadeh K, Regidor DL, McAllister CJ, Michael B, Warnock DG: Time-dependent associations between iron and mortality in hemodialysis patients. J Am Soc Nephrol 2005;16:3070-3080.

30 Kalantar-Zadeh K, Aronoff GR: Hemoglobin variability in anemia of chronic kidney disease. J Am Soc Nephrol 2009;20:479-487.

31 Meerson FZ, Evsevieva ME: Disturbances of the heart structure and function in chronic hemolytic anemia, their compensation with increased coronary flow, and their prevention with ionol, an inhibitor of lipid peroxidation. Adv Myocardiol 1985;5:201211.

32 Georgieva Z, Georgieva M: Compensatory and adaptive changes in microcirculation and left ventricular function of patients with chronic iron-deficiency anaemia. Clin Hemorheol Microcirc 1997;17:21-30.

33 Chen J, Gul A, Sarnak MJ: Management of intradialytic hypertension: the ongoing challenge. Semin Dialysis 2006;19:141-145.

34 Parfrey PS, Foley RN, Wittreich BH, Sullivan DJ, Zagari MJ, Frei D: Double-blind comparison of full and partial anemia correction in incident hemodialysis patients without symptomatic heart disease. J Am Soc Nephrol 2005; 16:2180-2189.

35 Ogawa T, Nitta K: Erythropoiesis-stimulating agent hyporesponsiveness in end-stage renal disease patients. Contrib Nephrol 2015;185: 76-86.

36 Wish JB: Assessing iron status: Beyond serum ferritin and transferrin saturation. Clin J Am Soc Nephrol 2006;1(suppl 1):S4-S8. 
37 Eschbach JW: The anemia of chronic renal failure: pathophysiology and the effects of recombinant erythropoietin. Kidney Int 1989; 35:134-148.

38 Vaziri ND: Safety issues in iron treatment in CKD. Semin Nephrol 2016;36:112-118.

39 Porto G, De Sousa M: Iron overload and immunity. World J Gastroenterol 2007;13: 4707-4715.
40 Gupta A, Zhuo J, Zha J, Reddy S, Olp J, Pai A: Effect of different intravenous iron preparations on lymphocyte intracellular reactive oxygen species generation and subpopulation survival. BMC Nephrol 2010;11:16.

41 Patruta SI, Edlinger R, Sunder-Plassmann G, Horl WH: Neutrophil impairment associated with iron therapy in hemodialysis patients with functional iron deficiency. J Am Soc Nephrol 1998;9:655-663.
42 Deicher R, Ziai F, Cohen G, Mullner M, Horl WH: High-dose parenteral iron sucrose depresses neutrophil intracellular killing capacity. Kidney Int 2003;64:728-736.

43 Ichii H, Masuda Y, Hassanzadeh T, Saffarian M, Gollapudi S, Vaziri ND: Iron sucrose impairs phagocytic function and promotes apoptosis in polymorphonuclear leukocytes. Am J Nephrol 2012;36:50-57. 